\title{
Gestion Des Risques Majeurs Au Maroc: Les Instabilités De Terrain
}

\section{Yazidi Mohamed}

Professeur Assistant, Laboratoire des sciences de la Terre Centre Régional des Métiers de l'Education et de la Formation, Marrakech-

Safi, Maroc

\section{Eloutassi Noureddine}

Professeur habilité, Laboratoire des sciences de la Vie

Centre Régional des Métiers de l'Education et de la Formation, Fès, Maroc

\section{Nabih Khadija}

Docteur en chimie, Laboratoire de Chimie Organique

Délégation du MENFP, AREF Marrakech - Safi, Maroc

\section{Hammi Abdel Hadi}

Professeur Assistant, Laboratoire des sciences de la Vie, Centre Régional des Métiers de l'Education et de la Formation, Fès, Maroc

\section{Yazidi Abdelaziz}

Professeur d'enseignement supérieur, Laboratoire de cartographie géologique, Ecole Nationale de l'Industrie Minérale (ENIM), Rabat, Maroc

\section{Benziane Fouad}

Professeur d'enseignement supérieur, Laboratoire de cartographie géologique, Ecole Nationale de l'Industrie Minérale (ENIM), Rabat, Maroc

\begin{abstract}
Instabilities of the ground is regarded as one of the most serious problems that affect many areas in Morocco. The study is important and beneficial because it concerns various sections of the Rif area. Indeed, certain zones which came at the top have been seriously affected. Disorders are numerous: destruction of homes, loss of agricultural land, and deterioration of infrastructure (roads, railways, and bridges). The aim of this study is focused on the zones where the road network generally is much damaged. This, however, causes a disturbance and even an interruption of the road traffic during stormy periods.

The detailed study of lithology, climatology, geomorphology, hydrology, and the slope of the studied zones allows us to highlight the characteristics of the unstable grounds. It also helped us to figure out that the rheological nature of
\end{abstract}


the ground plays a significant role in the characterization of the type of movement. The analysis of various movements which was found enables us to conclude that an instability of the ground depends on the conjunction on many factors, whether they are of provision or release. The most important factors causing the instabilities of the ground are: The alternation of rocks of nature; permeability and plasticity were well contrasted. The rugged relief and steep slopes. Geotechnical properties. Seismicity. Irregular rainfall. Geomorphology and land use. Anthropogenic action. Spatial distribution of the risk zones shows that the majority of the studied sections experience strong risks and medium risks levels.

Keywords: Ground instability, the Rif area, risk zones

\section{Résumé}

Les instabilités de terrain constituent un des plus graves problèmes qui affectent plusieurs régions au Maroc. Cette étude est importante et bénéfique, car elle concerne en particulier des sections de la région rifaine qui viennent en tête des zones gravement affectées. Les désordres sont nombreux: destruction des habitations, pertes de terres agricoles et détérioration des infrastructures (routes, chemins de fer et ponts) En effet, le réseau routier est généralement très touché, ce qui provoque une perturbation, voire, une interruption de la circulation routière au cours des périodes orageuses. L'étude détaillée de la lithologie, de la climatologie, de la géomorphologie, de l'hydrologie et de la pente des zones étudiées, nous a permis de mettre en évidence les caractéristiques des terrains instables et de constater que la nature rhéologique du terrain joue un rôle important dans la caractérisation du type d'instabilité. L'analyse des divers mouvements rencontrés nous a permis de conclure qu'une instabilité de terrain dépend de la conjonction de nombreux facteurs qu'ils soient de disposition ou de déclenchement. Les plus importants facteurs étudiés et qui causent des instabilités de terrain sont : L'alternance de roches de nature, de perméabilité et de plasticité très contrastées. La presence de relief heurté et des Pentes. Les propriétés géotechniques. La séismicité. La pluviométrie irrégulière. La géomorphologie et occupation du sol. L'action anthropique. La distribution spatiale des zones à risque, montre que la majorité des sections étudiées, passent par des niveaux à fort risque et à moyen risque.

Mots clés : Instabilités de terrain, la région rifaine, zones à risque

\section{Introduction}

Le Maroc est exposé aux phénomènes climatiques, météorologiques, géologiques ou biologiques. Ces phénomènes causent des risques majeurs 
tels: les inondations, les crues torrentielles, les séismes et les glissements de terrain. La conjoncture de ces phénomènes naturels provoque des dommages importants, voire compromettre le développement socio-économique des régions exposées aux catastrophes naturelles (Charte Nationale, 2015).

Dans cette étude nous nous sommes intéressés aux instabilités de terrain qui causent d'importants dégâts sur les infrastructures qui sont qualifié de risques majeur. Il s'agit de désordre qui implique de nombreux enjeux économiques et environnementaux.

Ainsi, les instabilités de terrain (écroulements, éboulements, solifluxions, glissements de terrain...) existent en certains points du globe. Leurs durées de manifestation sont variées : elles peuvent se traduire soit d'une façon brutale, en quelques minutes, soit s'étaler sur une longue période. Au Maroc, nombreuses sont les régions les plus touchées par ces instabilités de terrain. Ce problème, préoccupe les chercheurs (Gartet et al., 2009), vu le désordre qu'elles causent dans le réseau routier (Figure 1), les agglomérations, les terres agricoles. Elles peuvent être la cause de pertes humaines (INGÉROP, NOVEC, \& ACTERRA, 2015).

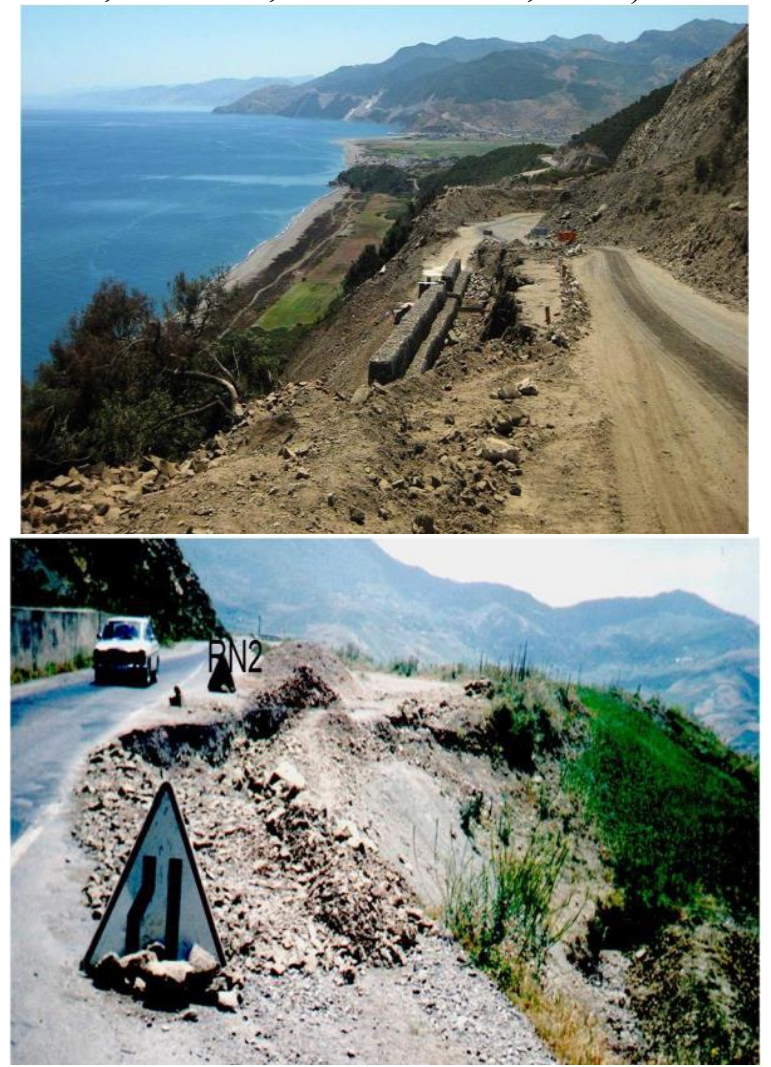

Figure 1 : Dégradation des routes marocaines à cause des instabilités de terrain 
Notre contribution est de chercher les causes et ensuite les solutions aux nuisances causées par certains types d'instabilités de terrain. C'est dans cette optique que nous comptons mener notre approche des instabilités en vue, d'abord, d'en définir les risques puis d'en délimiter les causes et enfin de proposer des solutions.

\section{Sélection des critères des instabilités de terrain}

L'instabilité de terrain constitue une famille très diversifiée de phénomènes qui intéressent le sol et le sous-sol. Elle est en relation avec l'évolution géodynamique. L'instabilité d'un terrain est due au changement des données qualitatives et quantitatives. Elle est liée, en particulier, à la nature et aux conditions géologiques, hydrologiques, géomorphologiques, séismiques, aux caractéristiques géotechniques des sols et des roches rencontrés, aux conditions météorologiques et à l'activité humaine (Yazidi, 2002).

\section{Classification et méthodologie}

Les mouvements de terrains correspondent à une rupture et à un déplacement des matériaux (Flageollet, 1989). Ces mouvements sont des phénomènes qui introduisent de nombreuses combinaisons possibles entre matériaux mis en œuvre et agents moteurs. Leur classification dépend étroitement de l'opinion de l'auteur quant à l'importance du poids de certains facteurs par rapport aux autres.

De nombreux auteurs se sont intéressés à la classification des mouvements de terrain. Dans notre étude, nous nous sommes basés sur la classification de Mougin (1973) adaptée et modifiée par Yazidi (2002), Yazidi et Benziane $(1995,2000)$ qui présentent une classification relativement facile à utiliser où ils intègrent l'existence de la forme de la surface de rupture. L'approche prend en compte les critères suivants: l'existence d'une surface de rupture; le degré de relation de cette surface avec le contexte géologique; le degré de remaniement de la masse glissée; la forme de la surface de rupture; la nature des terrains impliqués; la présence de niches d'arrachement et l'extension de la masse glissée.

\section{Techniques de l'étude des instabilités de terrain}

Les instabilités dépendent d'un certain nombre de facteurs. Yazidi et Benziane (1995) établissent des techniques d'étude. Ils proposent des tableaux schématiques qui permettent aux intéressés de connaître les principales causes génétiques des instabilités de terrain au Maroc (Tableau 1) ainsi que des relations qui peuvent exister entre les facteurs génétiques et les types de mouvement de terrain (Tableau 2). 
Tableau 1. Facteurs génétiques d'un glissement de terrain (Benziane \& Yazidi, 1995)

Augmentation (forces favorables au mouvement)

Augmentation de la pente ou de la hauteur du talus : par érosion, par mouvement tectonique, par intervention anthropique

Surcharge : par les agents naturels (eau, roches,...), par action anthropique (terrassement)

Ablation du support sous-jacent : par les agents naturels (érosion), par intervention humaine

Vibration et séismes.
Diminution de la résistance du matériau

Facteurs intrinsèques inhérents aux matériaux affectés : nature minéralogique et lithologique, topographie du talus ou du versant

Facteurs actifs en relation indirecte avec le matériau affecté : Modifications physicochimiques en relation avec les agents météoriques, augmentation des pressions interstitielles, modification de la structure intergranulaire, influence de la végétation, action anthropique

Le résultat de cette approche nous permet de montrer que les zones où les indices d'instabilités de terrain ont été observés, sont généralement considérées comme étant tout particulièrement vulnérables ou zones à instabilités déclarées. L'examen des instabilités déclarées dans la zone d'étude, se fait à partir des données qualitatives et quantitatives tirées de la géologie, de l'hydrologie, de l'hydrogéologie, de la climatologie et de la géomorphologie du site. Nous étudions dans ce qui suit certains facteurs qui pourront être les causes principales des instabilités rencontrées au Maroc.

Tableau 2. Les relations possibles entre les facteurs génétiques et les types de mouvements de terrain. (Benziane \& Yazidi, 1995)

\begin{tabular}{|c|c|c|c|}
\hline \multirow{2}{*}{\begin{tabular}{c} 
Cause du phénomène \\
\cline { 2 - 4 }
\end{tabular}} & Ecroulement & Glissement & Coulée \\
\hline $\begin{array}{c}\text { Entaille artificielle ou naturelle: } \\
\text { par érosion } \\
\text { par mouvement tectonique } \\
\text { par intervention anthropique }\end{array}$ & & & $*$ \\
\hline $\begin{array}{c}\text { Surcharge: } \\
\text { par les agents naturels (roches,...) } \\
\text { par intervention anthropique (travaux) }\end{array}$ & & $*$ & \\
\hline $\begin{array}{c}\text { Suppression du support sous-jacent: } \\
\text { par les agents naturels (érosion) } \\
\text { par intervention humaine }\end{array}$ & $*$ & $*$ & \\
\hline Augmentation des pressions interstitielles: & $*$ & $*$ & \\
\hline Vibrations et séismes & $*$ & & \\
\hline Augmentation des forces de gravité & $*$ & & \\
\hline Nature lithologique et minéralogique & $*$ & $*$ & $*$ \\
\hline Structure & $*$ & & \\
\hline Modification de la structure intergranulaire & & $*$ & \\
\hline Influence de la végétation & & $*$ & \\
\hline Action anthropique & & $*$ & $*$ \\
\hline Pluie d'orage (saturation) & & & $*$ \\
\hline Crue de rivière & & $*$ & \\
\hline
\end{tabular}




\section{Facteurs influençant les instabilités de terrain au Maroc} Le facteur climatique: la pluviométrie

Le Maroc appartient à la zone climatique semi-aride. L'influence des agents météorologiques agit directement ou indirectement sur le déclenchement des mouvements de terrain. Leur action s'exerce essentiellement sur la partie superficielle des terrains du fait des discontinuités stratigraphiques et tectoniques qui existent dans ces parties. Ces mouvements sont à l'origine des catastrophes naturelles affectant les vies humaines et les biens (Lemsioui, 2012).

La zone du Rif enregistre en moyenne 15 à 20 perturbations pluviométriques par an (Messouli, 2013). Ceci est dû au fait que l'air polaire froid qui accompagne ces perturbations après un long parcours vers le Sud, se réchauffe et perd généralement beaucoup de son activité lorsqu'il arrive aux latitudes du Maroc. Cependant, au moins une ou deux fois par an, l'air froid qui se caractérise par sa vigueur et sa rapidité lors de son expulsion, engendre des perturbations particulières qui se manifestent par des précipitations violentes.

Le climat de certaines régions du Rif, comme la région de Chefchaouene, est classé selon Sauvage (1963) et Emberger (1964) dans l'étage climatique sub-humide à humide. Il est caractérisé par une alternance de deux périodes bien différentes : une période de pluviosité (fin octobre début avril) suivie d'une période de sécheresse (fin avril - début octobre). En outre, un tel régime est sujet à de nombreuses variations, tantôt générales, tantôt locales. Cette zone, du Nord marocain, compte parmi les zones les plus pluvieuses du Maroc; elle reçoit plus de $1500 \mathrm{~mm} / \mathrm{an}$. Dans cette région le nombre de jours pluvieux est relativement élevé et dépasse 70 jours par an. La saison pluvieuse se caractérise par un maximum de précipitation aux mois de Décembre et Janvier. Cependant les précipitations peuvent être continues pendant plusieurs jours avec des intensités souvent très fortes.

A titre d'exemple, le 21 janvier 1996, journée que n'oublieront pas de sitôt les Jbalas, la quantité de pluies recueillies a représenté un record jamais atteint. En effet, ce jour-là, la pluviosité en 24 heures eut de $185 \mathrm{~mm}, 200$ $\mathrm{mm}$ et $210 \mathrm{~mm}$ respectivement à Chefchaoune, Bab Taza et Bab Berred. Ceci était dû à la persistance d'une dépression au voisinage du Maroc. L'hiver de l'année 2010 et le mois de février de l'année 2017 étaient aussi exceptionnelles, les précipitations étaient records. L'aggravation des phénomènes extrêmes englobe pluviométrie et périodes de sécheresse.

Les causes importantes du déclenchement des mouvements de terrain ne sont pas dues seulement à la quantité des pluies annuelles, mais surtout à la conjonction de l'intensité et du rythme des précipitations qui peuvent être très supérieures à la moyenne. 
L'étude minutieuse des différentes quantités de pluie ainsi que le recensement des différentes instabilités de terrain qui en découlent, nous permettent de remarquer que les mouvements de masse se déclenchent après une période de sécheresse qui agit sur le sol. La sécheresse est responsable de la variation du degré de saturation et de la perméabilité des couches superficielles (dessiccation). En effet, le sol subit des désagrégations et des fissurations, parfois sur plusieurs mètres de profondeur (suivant les plans de schistosité et de stratification). Lors des précipitations, les eaux de pluies s'infiltrent et saturent rapidement ce sol au niveau des surfaces moins résistantes (ou surfaces de glissement). Le manque de tapis végétal facilite aussi cet accroissement de l'infiltration. Plus les infiltrations augmentent en profondeur, plus les limites d'Atterberg (seuil de plasticité et de liquidité) sont franchies. Ce qui génère des solifluxions. Ce phénomène peut rester actif plusieurs mois après la fin de la saison humide (Yazidi \& Benziane, 2000; Yazidi, 2002; Messouli, 2013).

Dès lors, nous avons voulu savoir l'action directe des fortes précipitations. La question que nous pouvons nous poser :

Comment une précipitation produit-elle une instabilité? Pour répondre à cette question, il faut tenir compte de deux facteurs bien distincts. D'une part, ce sont les pluies abondantes et concentrées, très intenses, agressives et à fort ruissellement, arrivant le plus souvent en fin d'automne (en relation avec l'interférence polaire et tropicale), qui sont à l'origine des coulées boueuses, des coulées d'éboulis et des glissements superficiels. D'autre part, les pluies d'hiver, sont plutôt à l'origine des glissements profonds et généralisés du fait de leur abondance et de leur persistance (Lemsioui, 2012).

\section{Nature lithologique}

La nature lithologique des versants joue un rôle important dans les instabilités de terrain. Dans de nombreuses régions au Maroc, l'alternance des roches dures avec des niveaux tendres, constitue le premier élément responsable des glissements de terrain (Figure 2).

L'altération des roches joue également un rôle et induit des surfaces de discontinuité qui séparent la roche altérée de qualité mécanique médiocre de la roche saine. Ce cas est très répandu dans les nappes de flyschs rifaines (qui présentent une altération superficielle très accentuée) (Figure 3). En régions calcaires, l'altération provoque des phénomènes de karstification, ce qui peut engendrer des affaissements et des effondrements. 


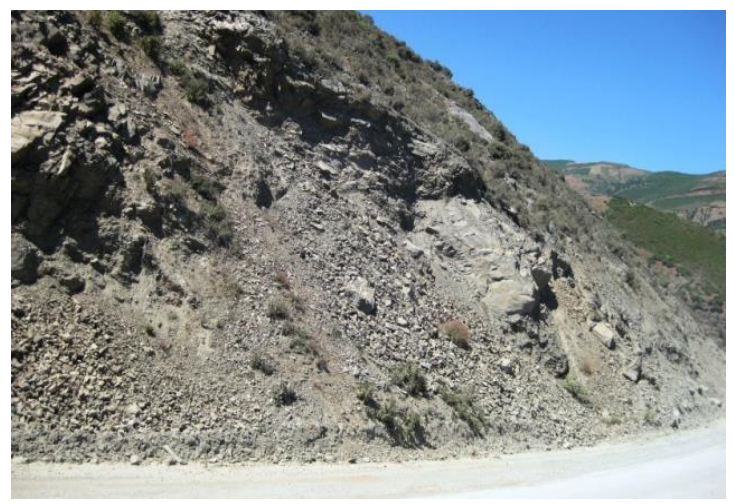

Figure 2. Alternance des roches dures avec des niveaux tendres

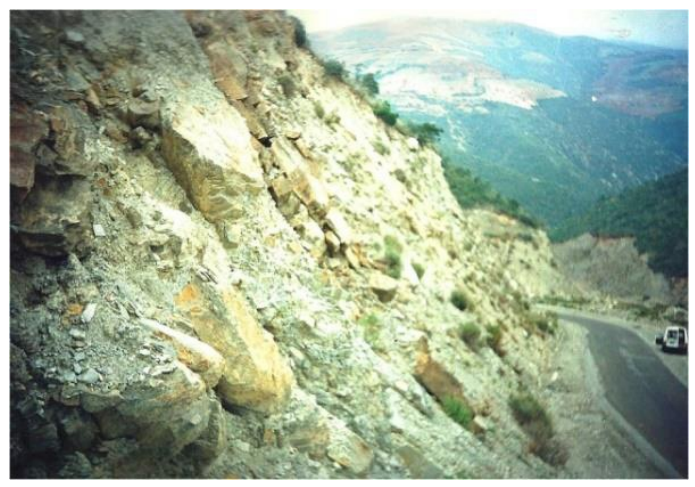

Figure 3. Flyschs rifaines sur les talus routiers

Les propriétés physico-chimiques de chaque type lithologique rencontré des zones étudiées se modifient au contact de l'eau. A titre d'exemple, une reptation lente ou des phénomènes de solifluxion sont conditionnés par l'approvisionnement en eau pluviale.

\section{Propriétés géotechniques}

Au Maroc, la majorité des instabilités de terrain sont caractérisées par un comportement plastique faisant intervenir le facteur eau qui joue un rôle primordial dans le déclenchement du phénomène. La plupart des terrains des zones d'étudiées longeant les routes sont constituées des marnes, des manteaux d'altération des marnes et des argiles. En présence d'une certaine quantité d'eau, la cohésion de ces matériaux diminue. Plus les roches sont imprégnées d'eau, plus la plasticité s'accroît. On peut même arriver au stade de liquidité ou liquéfaction (coulée boueuse) (Figure 4). 


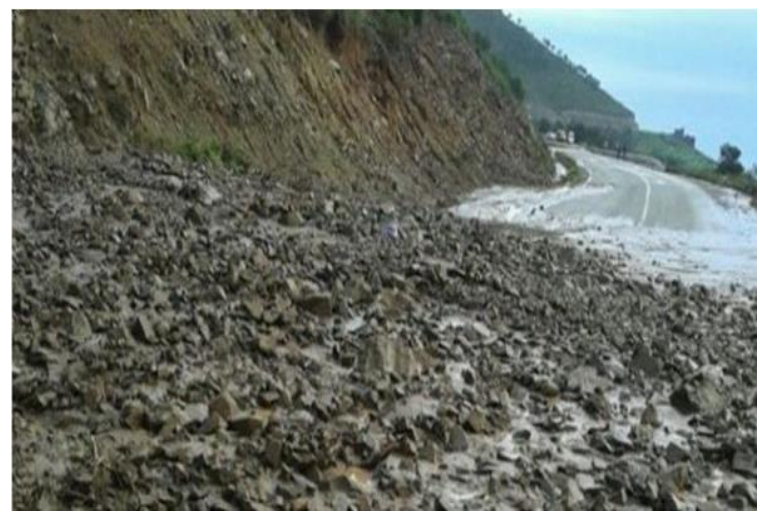

Figure 4. La rocade méditerranéenne bloquée par une coulée boueuse

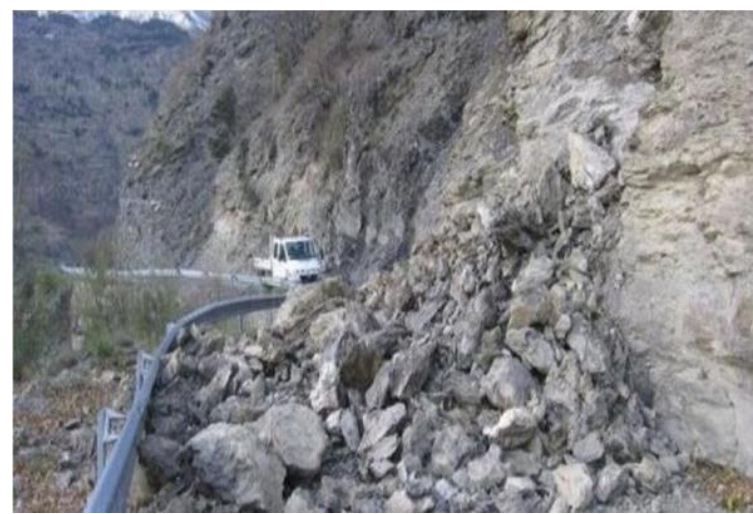

Figure 5. Eboulement rocheux a provoqué une interruption de la circulation routière

Les instabilités dans certaines régions peuvent être expliquées par les fortes pentes des talus marneux et argileux, leurs faibles caractéristiques physico-chimiques, la perte de cohésion et la désorganisation de la structure (minérale et architecturale). La direction et le pendage des couches, des plans de schistosité ou de fractures par rapport à la pente du versant jouent un rôle initial dans la genèse des surfaces de rupture et conditionnent le type des mouvements. Dans certaines zones du talus longeant les routes, les glissements de terrain sont dus à la structure du versant qui est conforme à la pente (Ezzardi et al., 2015). La densité de fracturation dans les massifs rocheux nous renseigne sur les hétérogénéités et sur les surfaces de discontinuités favorables à un écroulement ou à un éboulement (Figure 5). Les fractures ouvertes ou remplies par des matériaux argileux constituent, dans les massifs rocheux, des discontinuités favorables à divers types d'instabilités. Les multiples associations des différentes familles de discontinuités conduisent à une grande variété de mécanismes de ruptures 
élémentaires cinématiquement possibles: glissements plans, basculements, jeux de blocs.

\section{Séismicité}

L'activité sismique est considérée comme étant parmi les causes majeures des problèmes d'instabilité de terrain. En effet, c'est un facteur de perturbation régional de l'équilibre isostatique.

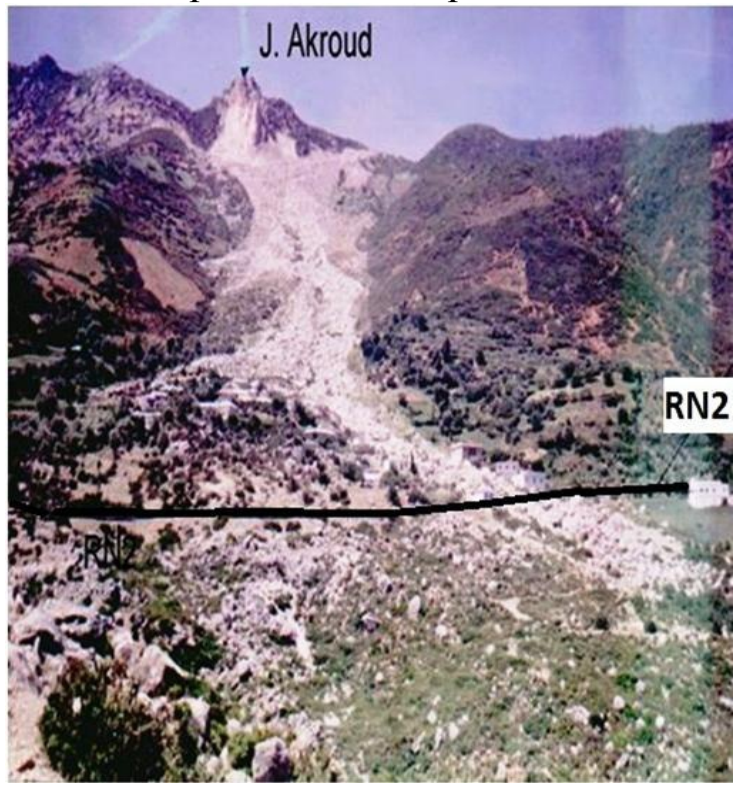

Figure 6. Les écroulements rocheux au niveau du Klippe de Jbel Akroud (RN2: route nationale 2)

Les réactivations d'instabilités de terrain se sont produites après des secousses sismiques (Figure 6). Ces instabilités se sont produites quelques jours après le séisme principal. Pour l'explication de ces phénomènes, certains auteurs (Seed \& Wilson, 1967; Moriwacki et al., 1985) démontrent que les séismes sont responsables de glissements de terrain provoqués par une réduction des contraintes effectives. Dans les pays marneux et argileux, les tremblements de terre peuvent produire des déformations retardées attribuées à la dissipation (ou dispersion) de surpressions interstitielles induites (ou appliquées) (Yashuara, 1995; Ezzardi et al., 2015).

\section{Géomorphologie et occupation du sol}

La plupart des mouvements de terrain résultent de la réactivation partielle d'anciennes instabilités et sont donc dues à un héritage géomorphologique. Ce dernier s'observe sur les versants où il se signale par des profils transversaux convexo-concaves à inclinaison prononcée et dont la longueur importante peut être continue ou discontinue. La géomorphologie des versants fournit des informations essentielles sur la dénudation 
continentale (Ezzardi et al., 2015). Elles constituent, par ailleurs, un préalable pour tout aménagement notamment dans les zones montagneuses. Les facteurs qui conditionnent la géomorphologie du bassin versant au Maroc sont surtout la perméabilité des terrains et l'énergie du relief. Ces derniers interfèrent de façon complexe.

\section{Pente}

Le degré de pente joue un rôle important dans la dynamique des versants. Il influence aussi bien l'hydrologie que l'hydrographie du bassin et donc des différentes instabilités qui affectent la région.

Dans certains secteurs, les perturbations de la topographie génèrent des contre-pentes, ce qui cause la stagnation des eaux et leur infiltration, réanimant, par conséquent, des instabilités de terrain qui touchent considérablement le réseau routier.

\section{Action anthropique}

Les interventions humaines modifient les paramètres naturels de manière spectaculaire: "Si les risques naturels échappent à la volonté de 1'homme, ils peuvent être aggravés par son action" (Ledoux B., 1995; Lemsioui, 2012; Messouli, 2013). Les principales actions anthropiques sont :

- le déboisement, les besoins en bois de chauffage, le surpâturage, les incendies et les extensions des zones cultivées font reculer la forêt;

- la population est en nombre toujours croissant. Les constructions envahissent le territoire, sans tenir compte des conditions géotechniques, ce qui cause dans certaines zones - surtout celles longeant les routes;

- une surcharge sur des talus à stabilité précaire. Cette surcharge génère et/ou réactive des glissements;

- le changement et le détournement de trajectoire des eaux. Ces actions sont surtout dommageables lorsque nous drainons les eaux perpendiculairement au versant, dans des canaux rarement bétonnés. A l'amont des glissements, l'irrigation augmente la charge et réactive les instabilités;

- 1'exploitation de manière aléatoire des matériaux par creusement de tranchées et de banquettes. Elle provoque des phénomènes de décompression des matériaux (d'où l'instabilité des versants).

On note encore:

- le poids des gabions et des murs de soutènement aggrave parfois la situation. En effet, ces systèmes provoquent une surcharge sur les versants du terrain naturel;

- la construction de certaines sections de routes, sur des talus métastables, diminue la capacité d'infiltration locale et élimine les voies naturelles de drainage; 
La modification des conditions du milieu naturel par l'action de l'homme peut déclencher ou aggraver les instabilités de terrain (Charte Nationale d'Aménagement du Territoire et du Développement Durable, 2015; INGÉROP, NOVEC, \& ACTERRA, 2015).

\section{La gestion du risque des instabilités de terrain au Maroc}

Nombreux sont les régions qui connaissent de fortes instabilités de terrain. Ces dernières affectent gravement et durablement les infrastructures, les agglomérations et leurs activités. Ces instabilités de terrain sont des phénomènes aux formes multiples. Elles représentent une évolution normale de versant, quoique d'apparence spectaculaire. Elles sont sporadiques et induisent une périodicité dans le transport solide.

La gestion du risque des instabilités de terrain est d'une importance capitale pour l'aménagement des régions qui sont soumises actuellement à une croissance urbaine rapide et souvent désordonnée.

Les nombreuses informations accumulées doivent être mises à la disposition de l'utilisateur, sous une forme synthétique, aisément compréhensible et exploitable. Ainsi, cette analyse débouche sur une carte interprétative, qui est la carte des risques. Une carte de ce genre, indique les aires d'instabilité ou à stabilité précaire et les zones stables.

Il est nécessaire de construire une banque de données sur les instabilités de terrain. Elle doit fournir les informations complètes. Ces informations doivent être claires, simplifiées, organisées, répertoriées, accessibles et consultables.

Elle permettra aux concernés d'envisager une stratégie de lutte contre les instabilités de terrain, proposera la réalisation de travaux confortatifs allant du simple entretien à la mise en œuvre de techniques de renforcement assez complexes, la prévention des risques et l'information du citoyen.

Nous proposons également la création d'un observatoire chargé du suivi. Cet observatoire permettra de diminuer les dégâts provoqués par l'instabilité de terrain et orienter les futures œuvres et les nouveaux travaux de construction dans les régions à risque tout en prenant en considération le paramètre d'instabilité.

\section{Conclusion}

Les instabilités de terrain sont des ensembles polygéniques qui résultent de l'action combinée de facteurs complexes. Les uns, facteurs permanents (la géologie, hydrogéologie, la topographie, la végétation...), créent les conditions indispensables aux instabilités de terrain; les autres (le rôle de l'eau, les agents climatiques, les séismes, les actions anthropiques...), dits facteurs dynamiques, agissent sous la dépendance des premiers et jouent le rôle de détonateur. 
Dans notre étude sur la recherche des différents facteurs, qu'ils soient causals de disposition ou causals de déclenchement, nous remarquons qu'il est le plus souvent illusoire de rechercher une cause unique à une instabilité de terrain. En effet, celle-ci dépend de la conjonction de nombreux facteurs d'instabilité.

Donc pour établir un diagnostique sur la stabilité d'un terrain ou sur les amplitudes des mouvements, il est nécessaire de dégager toutes les données qualitatives et quantitatives susceptibles d'entrer en jeu.

Certaines régions connaissent de fortes instabilités de terrain qui affectent gravement et durablement le réseau routier, les constructions et les activités. Ces instabilités de terrain sont des phénomènes aux formes multiples. Elles représentent une évolution normale de versant, quoique d'apparence spectaculaire. Elles sont sporadiques et induisent une périodicité dans le transport solide. C'est pourquoi, prévoir le risque de ces instabilités de terrain, c'est envisager la possibilité de leur déclenchement et de leur fréquence dans l'espace et dans le temps. Il en résulte que la prévision du risque nécessite une cartographie des zones à risque.

Ceci d'une part, d'autre part, La construction d'une banque de données et la création d'un observatoire chargé du suivi est fortement recommandé. L'existence de telles outils scientifiques aidera les professionnels et les centres de décisions à mieux raisonner leurs interventions sur le terrain particulièrement dans les zones isolées et dépourvues de moyens scientifiques et techniques appropriés.

\section{References:}

1. Charte Nationale d'Aménagement du Territoire et du Développement Durable (2015). Royaume du Maroc, ministère de l'aménagement du territoire, de l'urbanisme, de l'habitat et de l'environnement, aménagement du territoire et urbanisme, secrétariat général direction de l'aménagement du territoire.

2. Emberger, L. (1964). La position phytogéographique du Maroc dans l'ensemble méditerranéen. Al Awamia, Rabat, ${ }^{\circ}$ 12. pp. 1-15.

3. Ezzardi, A., Darif, J. \& Essami, A. (2015). La gestion cartographique de la dynamique des versants dans la zone de Beni Derkoul (Chefchaouen, Maroc). 2lème Rencontre des Géomorphologues Marocains, Milieux naturels atlantiques, géomorphologie et développement durable.

4. Fageollet, J.C. (1989). Les mouvements de terrain et leurs préventions. Collection géographie. Masson, Ed, 224p.

5. Gartet, A. \& Gartet, J. (2009). Gestion et prévention des risques des chutes de blocs et des éboulements rocheux à Sidi Harazem (région de Fès), Revue AFN Maroc, $\mathrm{N}^{\circ} 4-5$, pp 70-81. 
6. INGÉROP, NOVEC, \& ACTERRA (2015). Adaptation des routes au risque et au changement climatique au Maroc, Banque mondiale et direction des routes, Guide simplifié, 99279 v2, Maroc.

7. Ledoux, B. (1995). Les catastrophes naturelles en France. Ed. Payot et Rivages, Document Payot; 455p

8. Lemsioui, A. (2012). La prévention et la gestion des risques au Maroc, Guides et documents, Le Ministère de l'Energie, des Mines, de l'Eau et de l'Environnement, Royaume du Maroc.

9. Messouli, M. (2013). Anticipation et gestion des risques d'événements climatiques extrêmes; Etat des lieux sur des risques climatiques extrêmes et leurs impacts sur l'économie marocaine. Programme d'étude : Changement climatique, impact sur le Maroc et option d'adaptation globale. Ires (Institut Royal des Etudes Stratégiques).

10. Moriwacki, Y., Idrissi, I.M., Moses, T.L., \& Ladd, R.S. (1985). A reevaluation of the « $\mathrm{L}$ » Street slide in Anchorage during the 1964 Alaska earthquake. Comptes rendues XII ICSMFE, Rio de Janeiro, 3, pp 1583 - 1586.

11. Mougin, J.P. (1973). Les mouvements de terrain. Recherche sur les apports mutuels des études géologiques et mécaniques à l'estimation de la stabilité des pentes. Thèse, Univ. Grenoble, 295p.

12. Sauvage, C.H. (1963). Étage bioclimatiques. Notion et Carte au 1/200 000. Atlas du Maroc, Com. Géogr. Maroc. 44p.

13. Seed, H.B. \& Wilson, S.D. (1967). The Turnagain Heights landslide, Anchorage, Alaska. Journal of the Soil Mechanics and Foudation Division, ASCE, 93, pp 225 - 254.

14. Yashuara K. (1995). Consolidation and settlement under cyclic loading. Comptes rendues Int. Symp. On Compression and Consolidation of Clayey Soils, Hiroshima.

15. Yazidi, A. \& Benziane, F. (1995). Géologie du Rif - Relation avec les mouvements de terrain. Séminaire: Glissements de terrain: Cas du Nord marocain. Casablanca, Maroc.

16. Yazidi, M. (2002). Etude et cartographie de zones d'instabilités de terrain le long de la route nationale 2 entre Bab Taza et Bab Berred. Thèse Doc. National ; Uni. Ibn Tofail.

17. Yazidi, M. \& Benziane, F. (2000). Examen géologique de l'éboulement de la falaise du port de M'Diq (étude de stabilité). Rapport LPEE, Dossier N : 2000 / 08 / I / 097. 20p. et 18p. Annexe. 